\title{
A BOT-ENABLED APPROACH FOR WEB SERVICE DISCOVERY
}

\author{
Bahareh Zarei, Mahda Noura and Martin Gaedke \\ Technische Universität Chemnitz, Chemnitz, Germany
}

\begin{abstract}
With the wide adoption of service-oriented architectures, many service-based systems (SBSs) and mashups composed of Web services have been developed. Meanwhile, web service discovery, as a crucial component to guarantee the quality of Web service composition in SBS, gained increasing attention. Web service discovery aims to select the set of best service candidates according to the service requester's requirements. Existing syntactic and semantic-based approaches are proven to have low recall and precision or expecting high level of expertise from the service requesters. In this paper, we propose an approach for Web service discovery based on chatbots which obtains the best suited set of services to address the goals of service requesters. Chatbot-based interfaces are the emerging trend to provide a more natural human-device interaction. Compared to the existing web service discovery techniques, our approach alleviates the need for service requesters to have prior knowledge about the service location or its structure and enables them to identify services by leveraging natural language. We evaluated the approach through the collection of 56 queries from the test subject group on our repository of existing web service descriptions and report on quality metrics. The results shows high precision and recall.
\end{abstract}

\section{KEYWORDS}

Web Service Discovery, Service-Based Systems, Conversational interfaces, Natural Language Processing, Chat-Bot

\section{INTRODUCTION}

The web service paradigm has brought several advantages to software development and SOA-based applications. Providing simplicity by promoting reusability, agility and loose coupling are among the reasons for their widespread adoption in both academia and industry. The web services from major providers such as Google, Amazon and Facebook are published through public registries enabling developers to build loosely coupled applications (Zhang et al., 2018). The number of registered web services in ProgrammableWeb ${ }^{1}$ (PW) as of 2017 was reported more than 17,600 and this number has increased to almost 23,000 web services in just three years. Such services can be used to create service-based systems (SBS) or web compositions and mashups to offer value-added services (Zhang et al., 2018). One of the main challenges in SBSs is providing scalable and automated methods for selecting the relevant web services according to the service requester's requirements (Klusch, 2018). Web service discovery is a central component of service-based applications that can highly impact the effectiveness of web service compositions (Bhardwaj, 2015). Over the last decade a number of solutions have been proposed to address this challenge with respect to factors such as service description language or intended use of selected services (Klusch, 2018). The widely used techniques in the literature for Web service discovery are syntactic and semantic-based solutions. However, the existing solutions suffer in terms of low precision and recall (Zhang et al., 2018).

On the other hand, conversational interfaces as found in personal assistants such as Siri, Google Assistant, and Amazon Alexa represent a popular medium for users to interact with different smart devices and web services. Such interfaces provide a more natural method of interaction and a high level of usability for users (David Yoffie et al., 2018). Conversational agents and natural language interfaces, a.k.a. chatbots mimic human intelligence in conversations by reacting to the user's input through a realistic response.

${ }^{1}$ www.programmableweb.com 
According to Fuckner et al. (2013) enabling users to perform a task via natural language interaction can save up to $30 \%$ in time and $60 \%$ in the required effort. Using chatbots has proven to increase the retrieval accuracy (Abdul-Kader and Woods, 2015) and they can influence the user's behavior towards more informative interactions and thus more precise result.

The proposed solution by Fuckner et al. (2013) leveraged the idea of using chatbots to invoke desired web services. In this solution, a keyword-based matching is used. Despite the provided simplicity, keyword-based solutions are insufficient to discover all the relevant web services because users are assumed to not use the best keywords (in here best means the keywords already used in the web service description) to formulate the queries. To address this limitation several query expansion approaches have been proposed (Zhang et al., 2018, Ma et al., 2013). The goal of query expansion is to enhance the original query with the relevant concepts to increase the recall. Nevertheless, the proposed solutions either solely depend on the external knowledge bases such as ontologies or do not engage users for further communication.

To address the gaps of existing systems, we aim to design a chatbot able to understand the goal of service requesters (i.e., SBS and Web composition designers): The discovery chatbot receives textual utterances from service requesters as input, analyzes them using the query expansion technique to identify the domain and intended operations, generates reasonable responses according to our defined conversational flowchart and finally invoke the suitable web services. The contributions of this work are outlined as follows:

1. We proposed a method for creating a domain specific chatbot interface for web service discovery which is the first of its kind. The proposed approach is not reliant on the semantic descriptions of services and the requesters are not required to have prior knowledge about the service structure. Moreover, the approach integrates well-known query expansion techniques to extract the essential parameters from the requester's query and generate suitable follow up conversations.

2. We implement a prototype of the solution and conduct two sets of experiments to demonstrate its effectiveness for Web service discovery and query expansion.

The rest of the paper is organized as follows. In Section 2, our solution concept is discussed as well as an overview of the proposed architecture. To clarify the chatbot function a use case scenario is presented in Section 3 and Section 4 provides the study evaluation. The related work is reviewed in Section 5 and finally, Section 6 concludes the paper and provides future insights.

\section{DISCOVERY CHATBOT APPROACH}

In this section, we elaborate on the concept of our chatbot solution for web service discovery. The key requirements, design principles and overall architecture are discussed here.

\subsection{Concept}

Our solution is based on the bi-directional natural language communication between the service requester and the discovery chatbot. The envisioned chatbot is responsible for identifying the best web service candidates according to the requester's input. In the conventional input-intent-action-response conversation model (Baez and Daniel, 2019), the user expresses his intention as an input (e.g. "add a new appointment on July 4th, 5 p.m. to my calendar"). Then the chatbot extracts the user's intents using a Natural Language Understanding (NLU) unit (e.g. "add a new entry in the calendar") and maps it to an action that results in meeting the user's request ("addToCalander"). The execution of the action generates an output which will be used to produce a suitable response to the user (e.g." you have a meeting at the same time, do you want to reschedule?").

However, in our solution we use a customized version of the input-intent-action-response model to match web service discovery field. The customization is performed by refining the intent and action phases. The intent extraction involves identifying the key parameters of the desired web service (domain and operations (Zhang et al., 2018)) and the action refers to the matchmaking part to find the most relevant web services. Our solution is built on the following design principles:

- Discovery chatbot is a domain-specific chatbot that only handles the conversations regarding web services, therefore we use textual web service-related articles such as research papers as our dataset. All the steps of preprocessing were performed on the corpus which later transformed into numeric vectors. 
- Most of service descriptions are either XML-based (e.g. WSDL) or textual-based. In this work we use the textual description since it is more widely used specially in the case of APIs and RESTful web services.

\subsection{Architecture}

Figure 1 gives an overview of the discovery chatbot with its two main modules: The Natural Language Unit and Dialogue Manager. The service requesters can type in a description of the desired web service in the form of natural language text. This text will be analyzed by NLU to extract parameters such as target domain, operations, and entities. Such parameters are used to instantiate the Request Template. We define a Request Template as a generic objective description, in XML syntax, that is instantiated during run time. To address the problem of incomplete queries the chatbot includes the service requester in a conversation loop to provide the missing information such as domain and operations. The conversation flow and response generation are managed by the Dialogue Manager.

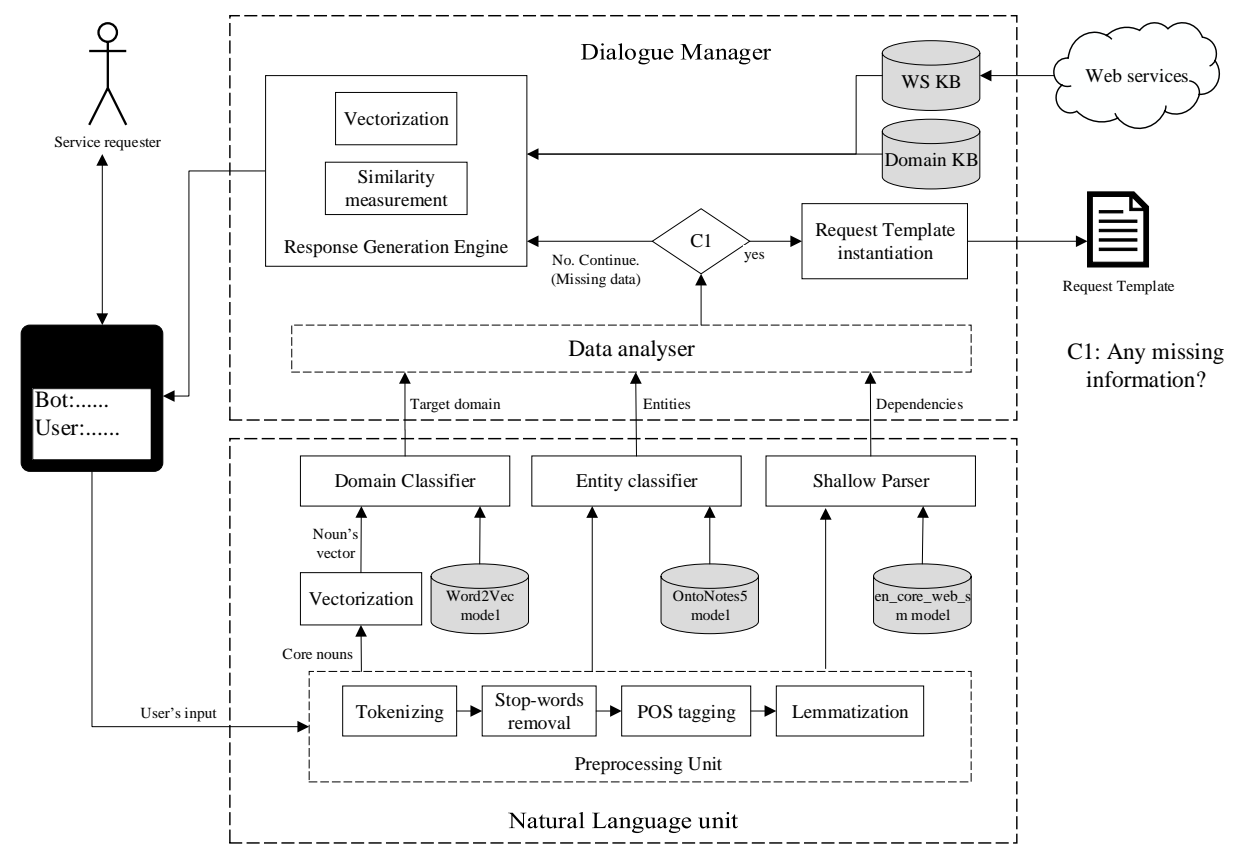

Figure 1. Overview of proposed approach

\subsubsection{Natural Language Unit}

The NLU consists of three main components: the Preprocessing Unit (PU), Classifiers and Shallow Parser. The PU pipeline is responsible for annotating the requester's query with tokens, removing stop-words, tagging each word with part-of-speech tags e.g. Nouns, Verbs, etc. and Lemmatization (Zarei and Gaedke, 2018). The output of PU is used as an input for classifiers. As defined in Abdul-Kader et al. 2015, classifiers are crucial components of chatbots responsible for normalizing the user's query and segmenting it into logical parts.

Domain Classifier determines the user's target domain. This way we efficiently reduce the search scope to potentially relevant services in that domain. As the first step we identified a set of words as representatives of each domain then upon every query the similarity among domain's representative words and the query's core nouns is calculated by the help of trained Word2vec model. The reason behind using query's core nouns is the greater importance of the core nouns in determining the domain. For example the two queries: "buy flight tickets" and "buy a house" refer to the different domains reflecting by core nouns "flight" and "house" 
(Zhang et al., 2018). To avoid missing some relevant services in the case of overlaps among two domains we consider both as target domains.

Entity classifier labels the entities with identifiers from the knowledge base. Entities are real-world objects such as locations, persons, countries, etc. For instance, the entities in the following sentence are labeled with respective identifiers: "find a Mark Twain (PERSON) book costs 1 \$30(MONEY)". Such entities will be used as web service parameters. The entity classifier uses spaCy ${ }^{2}$ library written in Python and a model trained on the OntoNotes5 corpus.

The next step is finding the requester's desired functionalities via Shallow parser. Each web service offers number of functionalities in the form of operations. To efficiently enhance the web service discovery, we have to take these operations into account. We defined the web service operation as $s f=<A V, A N,\left\{P_{i}, P_{o}\right\}>$ where $\mathrm{AV}$ denotes the action verb describing an operation and the AN as action noun that the operation effects on. The $P_{i}$ and $P_{o}$ are optional parameters for input and output. For instance, the query "Locate hotels in a given location" formulates as $s f=<$ locate, hotels, $\{$ GPS coordinates, -$\}\rangle$. The - here stands for an empty output. To extract operations from a given query we need to extract grammatical dependencies between pairs of words in the query. To obtain this, we used the spaCy dependency parser.

Not all the dependencies convey meaning with respect to service operations. Therefore, in this work, we use four main dependencies namely nsubj (nominal subject), dobj (direct object), iobj (indirect object) and prep (preposition) to extract the service operations. These dependencies are the core arguments in the English language according to Universal Dependencies ${ }^{3}$.

\subsubsection{Dialogue Manager}

Dialogue Manager is the chatbot's core component. Similar to the architecture presented by Raghuvanshi et al. 2018, it assesses the processed queries, applies the conversation's logic, and generates the best response. The parameters extracted from the query by NLU such as domain and operations are assessed by Data Analyzer for completeness and soundness. Based on the analysis result, the Data Analyzer triggers the Response Generation Engine to either ask the user for more detailed description or confirm the extracted parameters. The Request Template is only instantiated once both Data Analyzer and the requester confirm the parameters. By asking for the requester's confirmation we guarantee that the exact intended web services will be retrieved.

An important module of the Dialogue Manager is the Response Generation Engine (refer to Figure 1). The responses are generated according to the conversation flowchart in Figure 2. This flowchart describes the logic behind the conversations and orchestrates the dialogue's flow by predicting the user's behavior and generating the response accordingly.

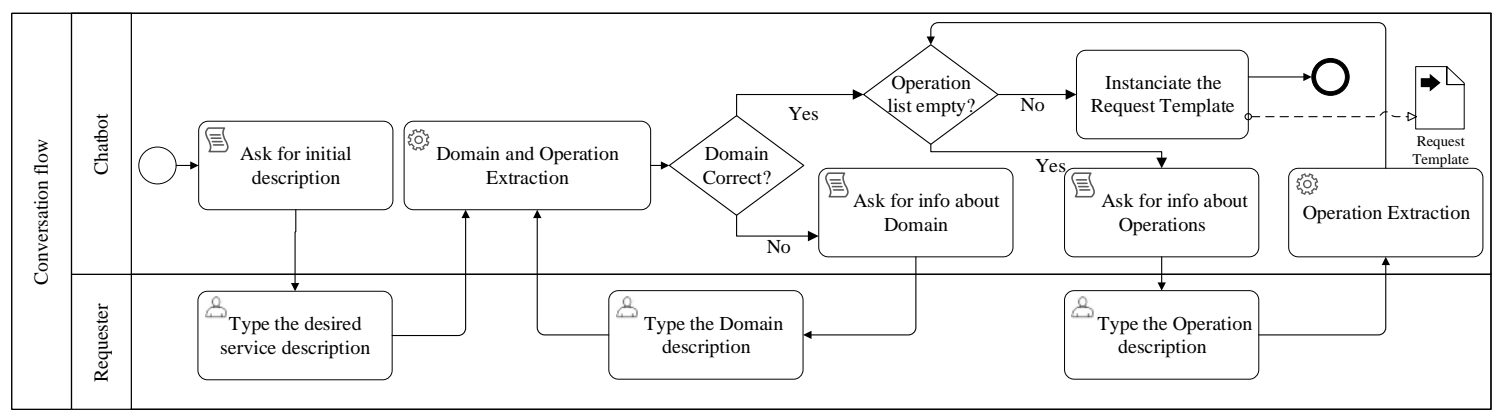

Figure 2. BPMN diagram for conversation flowchart

To further assist the requesters, the discovery chatbot is trained to respond to general queries about web services such as "What are the RESTful verbs?". The response's quality is highly dependent on the knowledge base used to train the chatbot. Developers can either write a database from scratch or make the chatbot learn from a textual corpus (Abdul-Kader and Woods, 2015). As already explained in the design

\footnotetext{
${ }^{2}$ https://spacy.io/

${ }^{3}$ https://universaldependencies.org/
} 
principles in Section 2, we developed a domain-specific training set as the chatbot knowledge base. To generate the best response, the TF-IDF (Term Frequency-Inverse Document Frequency) formula is used to calculate the similarity between the query $Q$ in document $D$ and the sentences in the training set:

$$
\mathrm{S}(\mathrm{Q}, \mathrm{D})=\sum_{w} t f_{w q} \cdot \frac{t f_{w D}}{t f_{w D}+\frac{k|D|}{a v g|D|}} \cdot \log \frac{|C|}{d f_{w}}
$$

The $t f_{w q}$ is the term frequency of word $w$ in $Q$. The summation indicates that the term's frequency has a direct impact on its importance. While the second part of the formula shows that the repetition is less important in the case of a short document.

The IDF component, in the end, gives higher weight to rare words compare to common words where $|C|$ is the total number of documents and $d f_{w}$ is the number of documents containing word $w$. This formula helps us to retrieve sentences as the potential response to the user's questions.

\section{AN EXAMPLE SCENARIO}

In this section, we walk through a use case scenario to demonstrate our approach in detail and highlight it's technical and architectural significance. A possible usage scenario for applying web services is in the context of Order Management Systems (OMS). Depending on the products, organizations use OMS to receive and process orders. Web services such as marketing, credit verification, billing, and financial services as well as shipment tracking are integrated to develop OMS. To further extend an existing OMS, the service requester plans to integrate the online tax calculation service. Such an API enables organizations to trade globally.

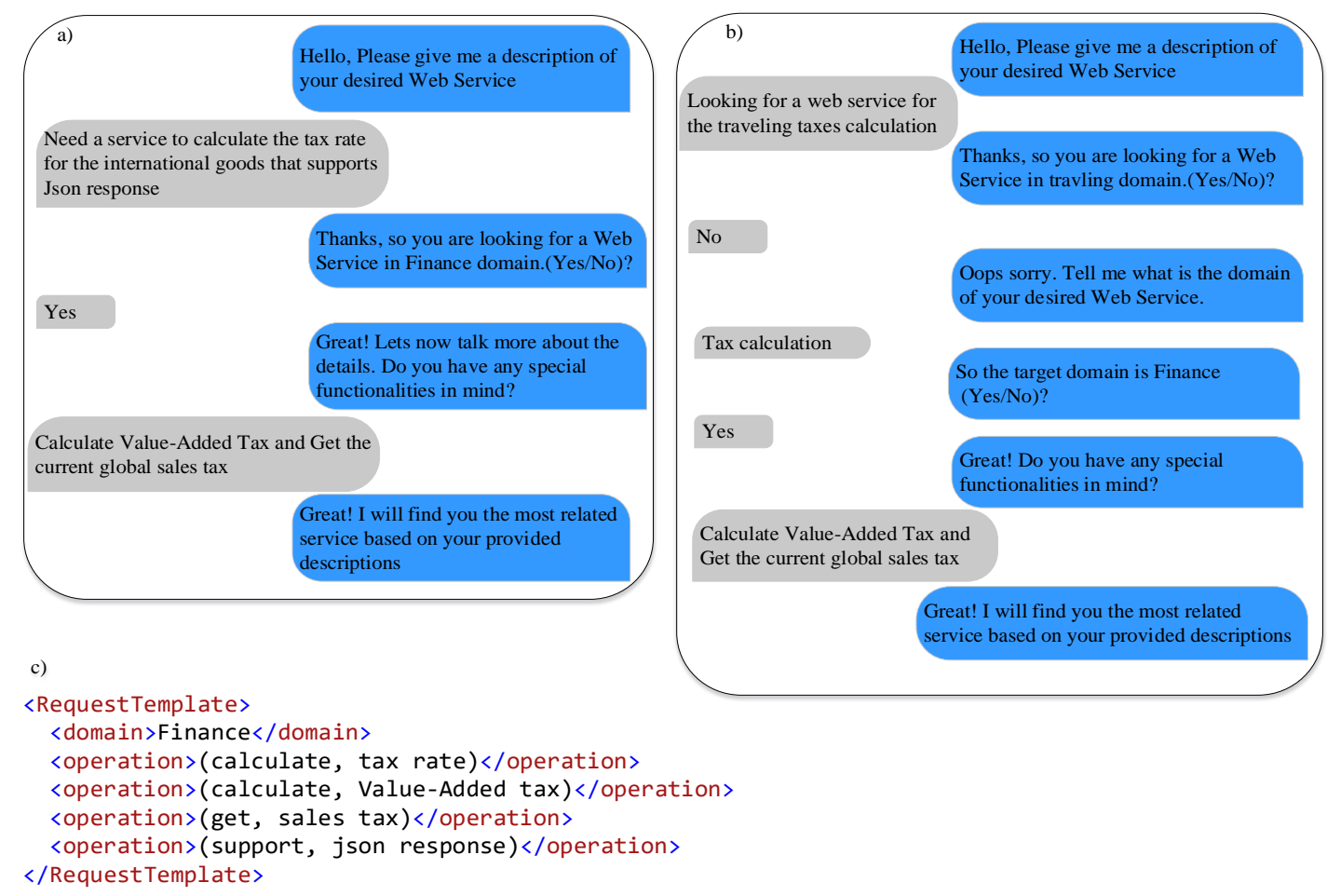

Figure 3. Example of various paths driven by chatbot. c) snippet of the Request template in XML syntax

Rounds of conversation among the service requester (gray boxes) and discovery chatbot (blue boxes) is presented in Figure 3. These examples are the two possible paths that conversation can be led to (based on the conversation flowchart in Figure 2). The chatbot is responsible for leading the user towards an informative response. The straightest forward path is when the user's initial description is informative 
enough (the domain and key operations are extractable. e.g. Figure 3 a). In the case of missing or wrongly extracted domain the chatbot keeps asking for more information until the requester confirms the domain and chatbot moves on to the operation extraction step (e.g. Figure $3 \mathrm{~b}$ ). The key operations are extracted from the initial description as well as follow up inputs. Extracted parameters are used to generate the Request Template as shown in Figure $3 \mathrm{c}$.

\section{EVALUATION}

We conducted two series of experiments to evaluate the NLU in terms of extracted domains and operations and calculate the precision and recall for retrieved web services. For this aim, we collected descriptive data of 1000 web services including tags and textual descriptions from the public PW registry. The web services are divided into 5 domains: Transportation, Mapping, Weather, Mobile, and Social. According to PW these categories are the most popular categories for web services and APIs. Popularity was measured as the ratio of the APIs have been used by mashups to all the existing APIs in that particular domain. We recruited eight subject requesters (including four developers, two postgraduate and two undergraduate students) to perform the two following tasks: 1) proposing ten words as domain representatives for each of the five target domains; and 2) constructing experimental queries to request web services in those domains. The final sets of representative words are built manually by selecting reasonable ones. Collectively 56 queries were constructed by our subject requesters. There was no limitation on the query length, and they range from short queries such as "extract GPS" to relatively long ones such as the queries in the use case scenario (Section 4).

\subsection{Evaluation Result}

For the first experiment the NLU output is evaluated. For each query the requesters verify the identified domain and the relevance of extracted operations. As already explained, we calculated the cosine similarity between the query's core nouns and each of the domain's representative vector, then the domain with highest similarity is chosen as the target domain. $98 \%$ of the identified domains for 56 queries matched with the intended domain. On the other hand, the operation relevance is determined based on a 0-3 scale ("0"- irrelevant, "1"-poorly relevant, "2"- relevant, " 3 "-highly relevant) and the evaluation result is shown in Table 1.

Table 1. NLU evaluation result

\begin{tabular}{cccc}
\hline Irrelevant & Poorly relevant & Relevant & Highly relevant \\
\hline $9 \%$ & $10 \%$ & $20 \%$ & $61 \%$
\end{tabular}

A thorough review determined that the irrelevant and poorly relevant operations (around 19\% of the total identified operations) are mainly derived from the general phrases in the query in the case of long descriptions. On the other hand, more relevant operations will be derived if we consider other types of dependencies such as propositions dependencies. This will be one of our directions for the future enhancement.

To evaluate the quality of discovery process, for each query we calculated the two parameters: precision and recall as follows:

$$
\text { Precision }=\frac{\left|W S_{r t} \cap W s_{r l}\right|}{W s_{r t}} \text { and Recall }=\frac{\left|W S_{r t} \cap W s_{r l}\right|}{W S_{r l}}
$$

where precision indicates the preciseness of the approach in discovering the intended web services and recall refers to the probability of a relevant document retrieval. The $W S_{r t}$ and $W S_{r l}$ in above formula denote the retrieved and relevant web services. The calculated precision and recall for our approach are respectively 0.78 and 0.80 . This result represents the good performance of our approach in discovering meaningful and relevant web services. 


\subsection{Threats to Validity}

In this section, we discuss the threats to our evaluation's internal and external validity. The internal validity concerns with the experiment's result repeatability. In our experiments, we relied on our subjects with different backgrounds to manually build the domain's representative set, construct the experiment queries, and evaluate the relativeness of the retrieved web service. A threat to internal validity can occur due to the subject's lack of familiarity with the selected domains and process. To resolve this threat, we used domain-related web pages to familiarize the subjects with the domains and described them the process step by step.

The external validity concerns about generalizing the study result to situations outside the context of this study. One external validity threat in this work concerns the APIs used for our experiments. We extracted the APIs from five popular domains in the public PW repository. Since this repository relies on the input APIs from API providers, the popularity and domain scales will change over time. Therefore, this will introduce a threat to domain selection validity. To overcome this threat, we plan to evaluate our work on multiple registries other than $\mathrm{PW}$.

\section{RELATED WORK}

Our work aims at providing a novel solution for web service discovery issue by leveraging chatbot technology. The number of works associated with chatbots is increasing every day and they cover different areas ranges from chatbot strategy design and response improvement to the variety of applications which they contribute to. This technology has a high evolving potential from one contribution to the next by adding new features (Abdul-Kader and Woods, 2015). To be able to justify the novelty of our work we review number of practices in the web service discovery field.

Existing discovery approaches can be categorized based on various metrics such as web service's functional and non-functional requirements or Quality of Service (QoS). From matchmaking point of view, the two dominant research directions are syntactic-based and semantic-based approaches (Mohebbi et al., 2010). The syntactic-based solutions like keyword matching and some of the recent enhancements such as clustering algorithms and WSDL structural matching are relatively easy nevertheless they mainly failed to fulfill the user's requirements in the terms of precision and recall (Bhardwaj, 2015, Zhang et al., 2018). The semantic-based approaches managed to overcome these drawbacks to some extent by proposing solutions in mainly two subcategories of logic and non-logic based (Zhang et al., 2018). In the logic-based solutions, a reasoning algorithm based on the domain ontology and semantic Web service description language (SAWSDL) is designed (Klusch, 2008). The discovery solutions can benefit from ontological knowledge with more precise results since the comparison is based on the semantic matching at the abstraction level. The good performance of such approaches has been proved, although the effort required for managing ontologies and service annotation is high (Bhardwaj, 2015, Mohebbi et al., 2010). The ontology heterogeneity is another issue, meaning the service provider and requester are not using a unique ontology. In such cases, coordination among ontologies should be done by ontology mapping algorithms (Mohebbi et al., 2010).

In one relevant study, Fuckner et al. 2013 proposed a dialogue-based multi-agent platform using the WSDL file as the only source of information. The key concepts from the user's request will be extracted and used to select and execute the exact web services. The concept matching is mainly keyword-based which the limitations were discussed earlier. On the other hand, as a non-logic-based solution, Zhang et al. 2018 proposed an approach that focuses on improving the user's query quality by presenting a novel goal-oriented query expansion based on the service goals. Authors utilized the Stanford parser to extract the linguistic structure of the sentences used in web service description to categorize services. Compared to this work, we propose a novel approach that leveraged the chatbot technology to retrieve the best set of web service candidates based on the user's query. We use the query expansion technique to extract the domain and operations. This way the approach performance in the terms of precision and recall will be increased. 


\section{CONCLUSION AND FUTURE WORK}

This paper proposed a novel web service discovery method by leveraging chatbot technology. The discovery process is performed based on conversations among the requester and the chatbot. Such conversations are built upon a conversation flowchart that leads users into more informative responses. With the help of NLP and query expansion techniques, the crucial parameters are extracted from the requester's responses that enable us to generate a Request Template as the representation of the desired web service. By integrating the chatbot with the web service discovery process, we improve the retrieval rate in the terms of precision and recall and provide a natural language interface for the requesters to retrieve the desired services.

During the last decade number of researches have been conducted on applying chatbots and conversational interfaces in different fields (e.g. healthcare, educational systems, and disability care). However, the idea of integrating chatbots into the service discovery process opens up new research directions in the area of SBSs, SaaS, and mashup. The chatbot technology can cover the wide range of applications in these areas such as bot-enabled mashups and web compositions for enhancing the end-user development or requirement extraction chatbot for SaaS applications. These examples could be topics for future research.

\section{ACKNOWLEDGEMENT}

This work has been supported by the ESF and the Free State of Saxony with grant number 1000235478.

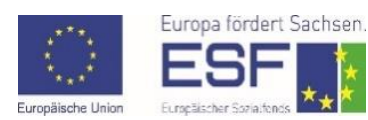

\section{REFERENCES}

Abdul-Kader, S. A. and Woods, J. (2015) 'Survey on Chatbot Design Techniques in Speech Conversation Systems', (IJACSA) International Journal of Advanced Computer Science and Applications, 6(7), pp. 72-80. Available at: www.ijacsa.thesai.org (Accessed: 30 December 2019).

Baez, M. and Daniel, F. (2019) 'Conversational Web Interaction: Proposal of a Dialog-Based Natural Language Interaction Paradigm for the Web', (October).

Bhardwaj, K. C. (2015) 'Machine Learning in Efficient And Effective Web Service Discovery', 14(3), pp. 196-214.

David Yoffie, P. B. et al. (2018) Voice War: Hey Google vs. Alexa vs. Siri. Available at: www.hbsp.harvard.edu. (Accessed: 6 January 2020).

Klusch, M. (2008) 'Semantic Web Service Description', CASCOM: Intelligent Service Coordination in the Semantic Web, pp. 31-57. doi: 10.1007/978-3-7643-8575-0_3.

Klusch, M. (2018) 'Service Discovery', in Alhajj, R. and Rokne, J. (eds) Encyclopedia of Social Network Analysis and Mining. New York, NY: Springer New York, pp. 2474-2484. doi: 10.1007/978-1-4939-7131-2_121.

Ma, S. P. et al. (2013) 'Web service discovery using lexical and semantic query expansion', Proceedings - 2013 IEEE 10th International Conference on e-Business Engineering, ICEBE 2013. IEEE, pp. 423-428. doi: 10.1109/ICEBE.2013.65.

Mohebbi, K. et al. (2010) 'A comparative evaluation of Semantic Web service discovery approaches', in iiWAS2010 12th International Conference on Information Integration and Web-Based Applications and Services. Paris, France, pp. 33-39. doi: 10.1145/1967486.1967496.

Raghuvanshi, A., Carroll, L. and Raghunathan, K. (2018) 'Developing Production-Level Conversational Interfaces with Shallow Semantic Parsing', in Proceedings of the 2018 Conference on Empirical Methods in Natural Language Processing: System Demonstrations. Brussels, pp. 157-162.

Zarei, B. and Gaedke, M. (2018) 'GOWDA: Goal-oriented Web Documents Querying tool', in DocEng '18 Proceedings of the ACM Symposium on Document Engineering. Halifax: ACM New York, NY, USA, p. No.47. doi: $10.1145 / 3209280.3229099$.

Zhang, N. et al. (2018) 'The Journal of Systems and Software Web service discovery based on goal-oriented query expansion', The Journal of Systems \& Software. Elsevier Inc., 142, pp. 73-91. doi: 10.1016/j.jss.2018.04.046. 